De Tampere à Séville : bilan de la sécurité européenne $(1 / 2)$

\title{
Version consolidée du Traité instituant la Communauté européenne (extrait)
}

\section{Communauté européenne}

\section{(2) OpenEdition}

\section{Journals}

Édition électronique

URL : http://journals.openedition.org/conflits/775

DOI : $10.4000 /$ conflits. 775

ISSN : $1777-5345$

Éditeur :

CCLS - Centre d'études sur les conflits lilberté et sécurité, L'Harmattan

Édition imprimée

Date de publication : 1 mars 2002

ISBN : 2-7475-3029-9

ISSN : 1157-996X

\section{Référence électronique}

Communauté européenne, « Version consolidée du Traité instituant la Communauté européenne (extrait) », Cultures \& Conflits [En ligne], 45 | printemps 2002, mis en ligne le 22 mars 2006, consulté le 30 mars 2021. URL : http://journals.openedition.org/conflits/775 ; DOI : https://doi.org/10.4000/ conflits. 775

Ce document a été généré automatiquement le 30 mars 2021.

Creative Commons License 


\title{
Version consolidée du Traité instituant la Communauté européenne (extrait)
}

\author{
Communauté européenne
}

TITRE IV (ex-titre III A)

VISAS, ASILE, IMMIGRATION ET AUTRES POLITIQUES LIÉES À LA LIBRE CIRCULATION DES PERSONNES

Article 61(ex-article 73 I)

Afin de mettre en place progressivement un espace de liberté, de sécurité et de justice, le Conseil arrête :

a) dans les cinq ans qui suivent l'entrée en vigueur du traité d'Amsterdam, des mesures visant à assurer la libre circulation des personnes conformément à l'article 14, en liaison avec des mesures d'accompagnement directement liées à cette libre circulation et concernant les contrôles aux frontières extérieures, l'asile et l'immigration, conformément à l'article 62 , points 2) et 3), et à l'article 63 , point 1), sous a), et point 2), sous a), ainsi que de mesures visant à prévenir et à combattre la criminalité, conformément à l'article 31, point e), du traité sur l'Union européenne ;

b) d'autres mesures en matière d'asile, d'immigration et de protection des droits de ressortis-sants des pays tiers, conformément à l'article 63 ;

c) des mesures dans le domaine de la coopération judiciaire en matière civile, visées à l'ar-ticle 65 ;

d) des mesures appropriées visant à encourager et à renforcer la coopération administrative visée à l'article 66 ;

e) des mesures dans le domaine de la coopération policière et judiciaire en matière pénale visant un niveau élevé de sécurité par la prévention de la criminalité et la lutte contre ce phénomène au sein de l'Union, conformément aux dispositions du traité sur l'Union euro-péenne.

Article 62(ex-article 73 J) 
Le Conseil, statuant conformément à la procédure visée à l'article 67, arrête, dans les cinq ans qui suivent l'entrée en vigueur du traité d'Amsterdam :

1) des mesures visant, conformément à l'article 14, à assurer l'absence de tout contrôle des personnes, qu'il s'agisse de citoyens de l'Union ou de ressortissants des pays tiers, lorsqu'elles franchissent les frontières intérieures ;

2) des mesures relatives au franchissement des frontières extérieures des Etats membres qui fixent:

a) les normes et les modalités auxquelles doivent se conformer les Etats membres pour effectuer les contrôles des personnes aux frontières extérieures ;

b) les règles relatives aux visas pour les séjours prévus d'une durée maximale de trois mois, notamment :

i) la liste des pays tiers dont les ressortissants sont soumis à l'obligation de visa pour franchir les frontières extérieures et de ceux dont les ressortissants sont exemptés de cette obligation ;

ii) les procédures et conditions de délivrance des visas par les Etats membres ;

iii) un modèle type de visa ;

iv) des règles en matière de visa uniforme ;

3) des mesures fixant les conditions dans lesquelles les ressortissants des pays tiers peuvent circuler librement sur le territoire des Etats membres pendant une durée maximale de trois mois.

Article 63(ex-article $73 \mathrm{~K}$ )

Le Conseil, statuant conformément à la procédure visée à l'article 67, arrête, dans les cinq ans qui suivent l'entrée en vigueur du traité d'Amsterdam :

1) des mesures relatives à l'asile, conformes à la Convention de Genève du 28 juillet 1951 et au Protocole du 31 janvier 1967 relatifs au statut des réfugiés ainsi qu'aux autres traités pertinents, dans les domaines suivants :

a) critères et mécanismes de détermination de l'Etat membre responsable de l'examen d'une demande d'asile présentée dans l'un des Etats membres par un ressortissant d'un pays tiers;

b) normes minimales régissant l'accueil des demandeurs d'asile dans les Etats membres ;

c) normes minimales concernant les conditions que doivent remplir les ressortissants des pays tiers pour pouvoir prétendre au statut de réfugié ;

d) normes minimales concernant la procédure d'octroi ou de retrait du statut de réfugié dans les Etats membres ;

2) des mesures relatives aux réfugiés et aux personnes déplacées, dans les domaines suivants :

a) normes minimales relatives à l'octroi d'une protection temporaire aux personnes dépla-cées en provenance de pays tiers qui ne peuvent rentrer dans leur pays d'origine et aux personnes qui, pour d'autres raisons, ont besoin d'une protection internationale ;

b) mesures tendant à assurer un équilibre entre les efforts consentis par les Etats membres pour accueillir des réfugiés et des personnes déplacées et supporter les conséquences de cet accueil ; 
3) des mesures relatives à la politique d'immigration, dans les domaines suivants :

a) conditions d'entrée et de séjour, ainsi que normes concernant les procédures de délivrance par les Etats membres de visas et de titres de séjour de longue durée, y compris aux fins du regroupement familial ;

b) immigration clandestine et séjour irrégulier, y compris le rapatriement des personnes en séjour irrégulier ;

4) des mesures définissant les droits des ressortissants des pays tiers en situation régulière de séjour dans un Etat membre de séjourner dans les autres Etats membres et les conditions dans lesquelles ils peuvent le faire.

Les mesures adoptées par le Conseil en vertu des points 3) et 4) n'empêchent pas un Etat membre de maintenir ou d'introduire, dans les domaines concernés, des dispositions nationales compatibles avec le présent traité et avec les accords internationaux.

Les mesures arrêtées en vertu du point 2), sous b), du point 3), sous a), et du point 4) ne sont pas soumises à la période de cinq ans visée ci-dessus.

Article 64(ex-article $73 \mathrm{~L}$ )

1. Le présent titre ne porte pas atteinte à l'exercice des responsabilités qui incombent aux Etats membres pour le maintien de l'ordre public et la sauvegarde de la sécurité intérieure.

2. Au cas où un ou plusieurs Etats membres se trouvent dans une situation d'urgence carac-térisée par un afflux soudain de ressortissants de pays tiers et sans préjudice du paragraphe 1 , le Conseil, statuant à la majorité qualifiée sur proposition de la Commission, peut arrêter au profit du ou des Etats membres concernés des mesures provisoires d'une durée n'excédant pas six mois.

Article 65(ex-article $73 \mathrm{M}$ )

Les mesures relevant du domaine de la coopération judiciaire dans les matières civiles ayant une incidence transfrontière, qui doivent être prises conformément à l'article 67 et dans la mesure nécessaire au bon fonctionnement du marché intérieur, visent entre autres à :

a) améliorer et simplifier :

- le système de signification et de notification transfrontière des actes judiciaires et extra-judiciaires ;

- la coopération en matière d'obtention des preuves ;

- la reconnaissance et l'exécution des décisions en matière civile et commerciale, y compris les décisions extrajudiciaires ;

b) favoriser la compatibilité des règles applicables dans les Etats membres en matière de conflits de lois et de compétence ;

c) éliminer les obstacles au bon déroulement des procédures civiles, au besoin en favorisant la compatibilité des règles de procédure civile applicables dans les Etats membres.

Article 66(ex-article $73 \mathrm{~N})$ 
Le Conseil, statuant conformément à la procédure visée à l'article 67, arrête des mesures pour assurer une coopération entre les services compétents des administrations des Etats membres dans les domaines visés par le présent titre, ainsi qu'entre ces services et la Commission.

Article 67(ex-article 73 0)

1. Pendant une période transitoire de cinq ans après l'entrée en vigueur du traité d'Amsterdam, le Conseil statue à l'unanimité sur proposition de la Commission ou à l'initiative d'un Etat membre et après consultation du Parlement européen.

2. Après cette période de cinq ans

- le Conseil statue sur des propositions de la Commission ; la Commission examine toute demande d'un Etat membre visant à ce qu'elle soumette une proposition au Conseil ;

- le Conseil, statuant à l'unanimité après consultation du Parlement européen, prend une décision en vue de rendre la procédure visée à l'article 251 applicable à tous les domaines couverts par le présent titre ou à certains d'entre eux et d'adapter les dispositions relatives aux compétences de la Cour de justice.

3. Par dérogation aux paragraphes 1 et 2 , les mesures visées à l'article 62 , point 2 ), sous b), littera i) et iii), sont, à compter de l'entrée en vigueur du traité d'Amsterdam, arrêtées par le Conseil, statuant à la majorité qualifiée sur proposition de la Commission et après consultation du Parlement européen.

4. Par dérogation au paragraphe 2 , les mesures visées à l'article 62 , point 2 ), sous b), littera ii) et iv), sont, après une période de cinq ans suivant l'entrée en vigueur du traité d'Amsterdam, arrêtées par le Conseil, statuant conformément à la procédure visée à l'article 251.

Article 68(ex-article 73 P)

1. L'article 234 est applicable au présent titre dans les circonstances et conditions suivantes : lorsqu'une question sur l'interprétation du présent titre ou sur la validité et l'interprétation des actes pris par les institutions de la Communauté sur la base du présent titre est soulevée dans une affaire pendante devant une juridiction nationale dont les décisions ne sont pas suscepti-bles d'un recours juridictionnel de droit interne, cette juridiction, si elle estime qu'une décision sur ce point est nécessaire pour rendre son jugement, demande à la Cour de justice de statuer sur cette question.

2. En tout état de cause, la Cour de justice n'est pas compétente pour statuer sur les mesures ou décisions prises en application de l'article 62, point 1), portant sur le maintien de l'ordre public et la sauvegarde de la sécurité intérieure.

3. Le Conseil, la Commission ou un Etat membre a la faculté de demander à la Cour de justice de statuer sur une question d'interprétation du présent titre ou d'actes pris par les institutions de la Communauté sur la base de celui-ci. L'arrêt rendu par la Cour de justice en réponse à une telle demande n'est pas applicable aux décisions des juridictions des Etats membres qui ont force de chose jugée.

Article 69(ex-article $73 \mathrm{Q}$ )

Le présent titre s'applique sous réserve des dispositions du protocole sur la position du Royaume-Uni et de l'Irlande et du protocole sur la position du Danemark et sans 
préjudice du protocole sur l'application de certains aspects de l'article 14 du traité instituant la Commu-nauté européenne au Royaume-Uni et à l'Irlande. (...)

INDEX

Mots-clés : droit européen, textes et documents officiels 